\title{
Fractionalization in a square-lattice model with time-reversal symmetry
}

\author{
B. Seradjeh, ${ }^{1}$ C. Weeks, ${ }^{1}$ and M. Franz ${ }^{1,2}$ \\ ${ }^{1}$ Department of Physics and Astronomy, University of British Columbia, Vancouver, BC, Canada V6T 1Z1 \\ ${ }^{2}$ Kavli Institute for Theoretical Physics, University of California, Santa Barbara, CA 93106
}

\begin{abstract}
We propose a two-dimensional time-reversal invariant system of essentially non-interacting electrons on a square lattice that exhibits configurations with fractional charges $\pm e / 2$. These are vortex-like topological defects in the dimerization order parameter describing spatial modulation in the electron hopping amplitudes. Charge fractionalization is established by a simple counting argument, analytical calculation within the effective lowenergy theory, and by an exact numerical diagonalization of the lattice Hamiltonian. We comment on the exchange statistics of fractional charges and possible realizations of the system.
\end{abstract}

Introduction.--It is now well-known that fractional quantum numbers can arise as the collective excitations of a manybody system. The canonical example of such fractionalization is a $2 \mathrm{D}$ electron gas (2DEG) placed in a transverse magnetic field in the fractional quantum Hall regime. At odd inverse filling factors, $v^{-1}>1$, the many-body ground state is described by a strongly-correlated Laughlin wave function and the time-reversal symmetry is broken. The excitations carry the fractional charge $v e$ (Ref. 1) and exhibit fractional (Abelian) statistics. $\stackrel{2}{=}$ The search for other systems that exhibit fractionalization is ongoing. Two important questions in this search are whether strong correlations or a broken timereversal symmetry is necessary for fractionalization to happen.

Recently the answer to both questions is argued to be negative. A group including the present authors ${ }^{-3}$ proposed a system with fractionally charged, anyonic excitations that can be described by a weakly-interacting wave function found by filling a set of single-particle states. Moreover, Hou, Chamon and Mudry ${ }^{4}$ have argued that a vortex in the Kekulé modulations of the hopping amplitudes on a honeycomb lattice, like that of graphene, binds a fractional charge $e / 2$ without breaking the time resversal symmetry.

In this paper we propose a system on a square lattice with time-reversal symmetry that exhibits fractionalization. The system consists of a square lattice threaded by one half of a magnetic flux quantum $\Phi_{0}=h c / e$ per plaquette on which electrons can hop to nearest-neighbor sites with no interaction. Time-reversal symmetry is preserved in this lattice model because electrons cannot detect the sign of the flux with magnitude $\Phi_{0} / 2$. In addition, we assume a dimerized modulation of hopping amplitudes as depicted in Fig. 1(a). Such modulations can arise as a Peierls distortion of a uniform ion lattice above a critical value of the electron-phonon coupling or, as discussed in Ref. 4 in the context of graphene, as an interaction-driven instability. We show that a vortex in the complex scalar order parameter describing this dimerization pattern generates a zero-energy bound state in the spectrum of electrons and carries a fractional charge. In addition to similar arguments to those of Ref. 4, we present a simple electron counting argument $t^{5.6}$ as well as numerical evidence demonstrating this effect. Importantly, we find that the fractionalization survives essentially intact beyond the low-energy theory of Ref. 4 in the presence of the lattice. We also show that the same pattern of fractionalization occurs for the $Z_{4}$ and $\mathrm{U}(1)$

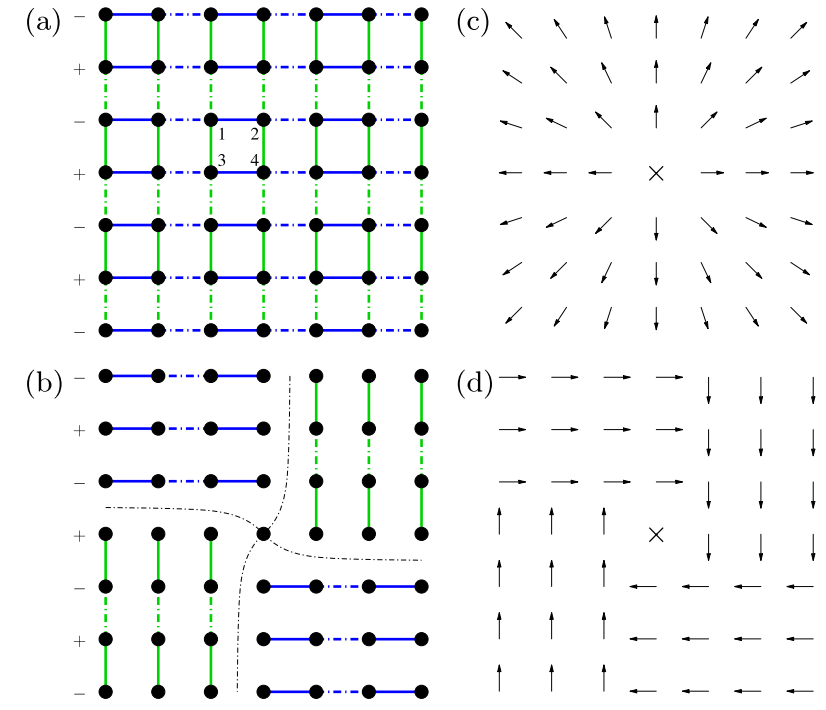

FIG. 1: (Color online) The model: (a) Square lattice with $\frac{1}{2} \Phi_{0}$ magnetic flux per plaquette and dimerized hopping amplitudes. The \pm on the left for each row show the choice of gauge for the Peierls phase factors. The solid (dashed) bonds indicate an increased (decreased) hopping amplitude in the $\hat{x}$ (blue) and $\hat{y}$ (green) directions as explained in the text. The four sites of the unit cell are marked. (b) The $Z_{4}$ vortex. The dashed lines indicate the domain walls sharing the center of the vortex. The phase of the local dimer order parameter $f_{i}$ (see text) around (c) the $\mathrm{U}(1)$ and (d) the $Z_{4}$ vortex. The $\times$ shows the center of each vortex where $f=0$.

vortices and clarify the issue of their confinement. We then give a brief discussion of the energetics of the Peierls distortion and touch upon possible experimental realizations of the model in artificially engineered semiconductor heterostructures and optical lattices.

The model.-We consider a square lattice with a tightbinding Hamiltonian

$$
\mathcal{H}_{e}=-\sum_{\langle i j\rangle}\left(t_{i j} e^{\mathrm{i} \theta_{i j}} c_{j}^{\dagger} c_{i}+\text { h.c. }\right)+\sum_{i} \epsilon_{i} c_{i}^{\dagger} c_{i},
$$

for electrons annihilated at site $\mathbf{r}_{i}$ by $c_{i}$. We assume the spins of all electrons are polarized along the field. Here, $t_{i j}$ are hopping amplitudes between nearest-neighbor sites and $\epsilon_{i}$ is the on-site potential. The magnetic field is included through the Peierls phase factors, $\theta_{i j}=\left(2 \pi / \Phi_{0}\right) \int_{\mathbf{r}_{i}}^{\mathbf{r}_{j}} \mathbf{A} \cdot d \mathbf{l}$. Each plaquette 
is threaded by flux $\Phi_{0} / 2$. We choose to work in the Landau gauge $\mathbf{A}=\left(\Phi_{0} / 2\right)(-y, 0)$ where we have set the lattice spacing to unity. The hopping amplitudes in our model form a dimerized pattern as shown in Fig. 1 (1) with alternating bonds $t_{i, i+\hat{x}}=t\left(1 \pm m_{x}\right)$ and $t_{i, i+\hat{y}}=t\left(1 \pm m_{y}\right)$. In the following we assume uniform $\epsilon_{i}=\epsilon$.

We can now arrange the four sites of the unit cell into a spinor field

$$
\psi_{\mathbf{k}}=e^{\frac{\pi}{4} \gamma_{2}}\left(c_{1 \mathbf{k}}, c_{2 \mathbf{k}}, c_{3 \mathbf{k}}, c_{4 \mathbf{k}}\right)^{T}
$$

to write the Hamiltonian as

$$
\mathcal{H}_{e}=\sum_{\mathbf{k} \in \mathrm{BZ}} \psi_{\mathbf{k}}^{\dagger}\left(\epsilon-2 t H_{\mathbf{k}}\right) \psi_{\mathbf{k}}
$$

in the reduced Brillouin zone BZ $=\left\{\mathbf{k}|| k_{x}|,| k_{y} \mid \leq \frac{1}{2} \pi\right\}$ with

$$
H_{\mathbf{k}}=\gamma_{0}\left(\gamma_{1} \cos k_{x}+\gamma_{2} \cos k_{y}+m_{x} \sin k_{x}+\mathrm{i} m_{y} \gamma_{5} \sin k_{y}\right) .
$$

We are using the standard Weyl representation of Dirac matrices: $\gamma_{0}=\sigma_{1} \otimes \mathbb{1}, \vec{\gamma}=\mathrm{i} \sigma_{2} \otimes \vec{\sigma}$ and $\gamma_{5}=-\mathrm{i} \gamma_{0} \gamma_{1} \gamma_{2} \gamma_{3}=\sigma_{3} \otimes \mathbb{1}$. The spectrum of $H_{\mathbf{k}}$ is given by

$$
E_{\mathbf{k}}= \pm \sqrt{\cos ^{2} k_{x}+\cos ^{2} k_{y}+m_{x}^{2} \sin ^{2} k_{x}+m_{y}^{2} \sin ^{2} k_{y}},
$$

which is doubly degenerate and symmetric: if $\psi_{E}$ is an eigenstate with energy $E$, i.e. $H_{\mathbf{k}} \psi_{E}=E \psi_{E}$, then $\gamma_{0} \gamma_{3} \psi_{E}$ is an eigenstate with energy $-E$ since $\left\{H_{\mathbf{k}}, \gamma_{0} \gamma_{3}\right\}=0$.

Low-energy theory.-The undistorted system with $\mathbf{m} \equiv$ $\left(m_{x}, m_{y}\right)=0$ has Dirac nodes at $k_{x}=k_{y}= \pm \pi / 2$ where the two energy bands meet. There is one node per reduced Brillouin zone. At nonzero constant dimerization $\mathbf{m}$ a gap $m=\left(m_{x}^{2}+m_{y}^{2}\right)^{1 / 2}$ opens up in the spectrum. We will focus our attention on the low-energy excitations of the system in the situation when the dimerization vector $\mathbf{m}$ is slowly varying in space. Using a polar representation for the hopping anisotropy $m_{x}+\mathrm{i} m_{y}=m e^{\mathrm{i} \chi}$ and by linearizing $\mathcal{H}_{e}$ around the nodes in the continuum limit we find the low-energy Hamiltonian

$$
H_{\mathbf{x}}=\gamma_{0}\left(\mathrm{i} \gamma_{1} \partial_{x}+\mathrm{i} \gamma_{2} \partial_{y}+m e^{\mathrm{i} \chi \gamma_{5}}\right)
$$

An interesting situation arises when a $\mathrm{U}(1)$ vortex is present in the dimerization vector $\mathbf{m}$. Such a vortex is characterized by a $2 n \pi$ winding in the phase $\chi$, with $n$ an integer, along a contour enclosing the vortex center. The continuum Dirac Hamiltonian (5) in this vortex background field is the same as that studied in Refs. 4 and 7. It admits $|n|$ degenerate zeroenergy bound states. $\stackrel{8}{=}$ This result has its origin in an index theorem for the Dirac Hamiltonian in topologically nontrivial background fields?

For a singly quantized vortex $(|n|=1)$ there is a single zero mode with support on one of the sublattices depending on the sense of the vortex. A standard calculation ${ }^{4.10}$ can be used to show that when the lattice is half-filled the charge carried by such a vortex is fractionalized, namely $\pm e / 2$, depending on whether or not the zero mode is occupied by an electron.

In our square lattice model one can also give a simple intuitive counting argument for the charge fractionalization that generalizes the construction deployed in the case of polyacetylene by Goldstone and Wilczek $\frac{5}{=}$ Consider a $Z_{4}$ vortex, depicted in Fig. 1(b,d), in the limit of strong dimerization, $m \simeq 1$. In this limit each thick bond in Fig. 1(b) can be thought of as representing one electron resonating between the two lattice sites. The topological structure of this $\mathrm{Z}_{4}$ vortex guarantees that in its core there is necessarily a site with no partner ${ }^{11}$ This site can be either occupied or empty, thus exhibiting $e / 2$ surplus or deficit of charge compared to the background, which has $e / 2$ per site on average. The $Z_{4}$ vortex can be adiabatically deformed into the $U(1)$ vortex of Fig. 1 (b) and to the weakly dimerized phase at $m \ll 1$. This indicates that the charge fractionalization persists in that limit, as explicitly verified by the analytical calculation ${ }^{4,10}$ and by the numerical results reported below. We note that this argument works in our model only in the presence of the magnetic flux, which is needed for the existence of the Dirac nodes in the spectrum. At zero flux there is a Fermi surface for any small $m$ and the unpaired center of a vortex does not create a bound state.

Numerics. - We have performed exact diagonalization of the tight-binding Hamiltonian (11) with different vortex configurations on lattices of size up to $52 \times 52$. Open boundary conditions were assumed in all cases. We consider two realizations of the vortex, a $Z_{4}$ vortex and a lattice version of the continuum $\mathrm{U}(1)$ vortex studied in the low-energy theory. To quantify the vortex structure we use a local dimer order parameter $^{12}$ defined at site $\mathbf{r}_{i}=\left(x_{i}, y_{i}\right)$ as

$$
f_{i}=\sum_{\hat{\mu}} \eta_{i, i+\hat{\mu}} t_{i, i+\hat{\mu}} .
$$

Here the $\hat{\mu}$ are the four nearest-neighbor unit vectors, $\eta_{i, i+\hat{x}}=$ $(-1)^{x_{i}}, \eta_{i, i+\hat{y}}=\mathrm{i}(-1)^{y_{i}}$ and $\eta_{i, i-\hat{\mu}}=\eta_{i-\hat{\mu}, i}$. (See also Fig. 2 in Ref. 12.)

The $Z_{4}$ vortex occurs if the energetics of the problem allow only dimerizations along the high-symmetry directions of the crystal, e.g. $\mathbf{m}=( \pm m, 0)$ and $(0, \pm m)$. A vortex is then obtained by putting together four possible domains as illustrated in Fig. 1(b), separated by domain walls emanating from the vortex center. The phase of the order parameter for the $Z_{4}$ vortex is depicted in Fig. 11d).

The U(1) vortex occurs if the energy of the state characterized by dimerization vector of the form $\mathbf{m}=m(\cos \chi, \sin \chi)$ is approximately independent of $\chi$. We construct the $\mathrm{U}(1)$ vortex on the lattice by discretizing the continuum vortex field $\varphi(z)=z /|z|$ where $z=x+\mathrm{i} y$ is the complex coordinate of the point $(x, y)$. Explicitly,

$$
t_{i, i+\hat{\mu}}=t_{0}\left(1+m \operatorname{Re}\left[\eta_{i, i+\hat{\mu}}^{*} \varphi\left(\mathbf{r}_{i}+\frac{1}{2} \hat{\mu}\right)\right]\right),
$$

where the asterisk denotes the complex conjugation. Fig.1 (b) shows the phase of $f_{i}$ clearly illustrating the $\mathrm{U}(1)$ form. The $\mathrm{U}(1)$ structure can be extended to study the case for multiple vortices and antivortices. For vortices at $z_{1}, z_{2}, \ldots$ and antivortices at $z_{1}^{\prime}, z_{2}^{\prime}, \ldots$ this is achieved by taking a product of vortex fields $\varphi(z)=\prod_{a}\left(z-z_{a}\right) /\left|z-z_{a}\right| \prod_{b}\left(z-z_{b}^{\prime}\right)^{*} /\left|z-z_{b}^{\prime}\right|$ in Eq. (7).

A typical sample of our results for a system with one and two vortices is shown in Figs. 2] and3, respectively. The structure of the vortex is reflected in the symmetries of the zero 
(a)

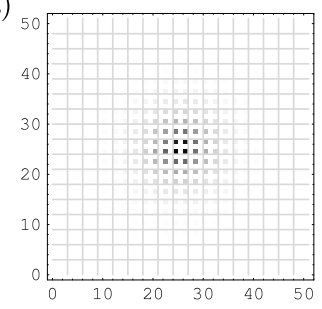

(c)

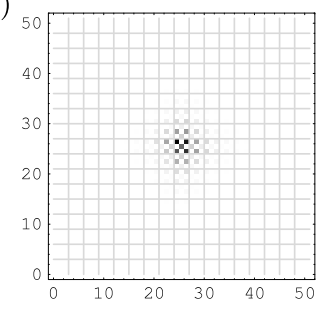

(b)

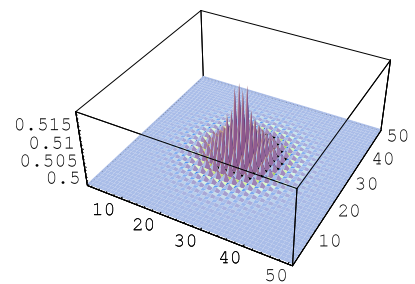

(d)

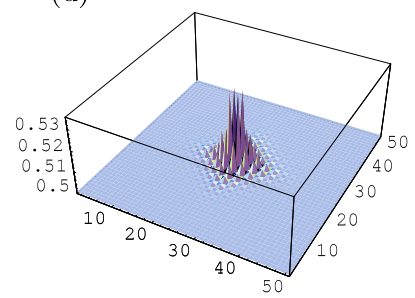

FIG. 2: (Color online) The charge density at half-filling with zero mode filled in the $\mathrm{U}(1)$ vortex with $m=0.15(\mathrm{a}, \mathrm{b})$. The same plots for the $Z_{4}$ vortex and $m=0.30(\mathrm{c}, \mathrm{d})$. The lattice size $51 \times 51$ and the gridline spacing is 3 . The top-view density plots on the left are included to show the symmetry of the solution for each vortex and the support on a single sublattice.

mode and the corresponding charge density at half-filling. For a single vortex, Fig. 2, the charge density at half-filling with the zero mode filled shows an excess charge localized at the vortex above the background value. For both the $\mathrm{U}(1)$ and $Z_{4}$ vortex this excess charge integrates to $0.5 e$ to within machine accuracy confirming the existence of the fractional charge. Remarkably, the charge density of the half-filled system with the $Z_{4}$ vortex is essentially the same as that with the $U(1)$ vortex. Especially, no charge is bound to the domain walls far away from the center.

The system with two vortices has two zero-energy states with support on the same sublattice, Fig. 3 a). It is important to note that, as illustrated in Fig. 3 b), these remain exact zero modes even when the intervortex separation $d_{\mathrm{vv}}$ becomes short compared to the size of the bound state wavefunction $\xi \sim m^{-1}$. The zero modes are topologically protected in accordance with the index theorem of Ref. 9 which states that the number of exact zero modes in the system is bounded from below by the total vorticity of the order parameter $f$. This can be understood on a more intuitive level by noting that since the two bound states reside on the same sublattice the matrix element of the Hamiltonian (1) with $\epsilon=0$ between the two states vanishes identically and hence leads to no splitting in energies. Alternatively, one can consider a process of merging $n$ single vortices into one vortex with winding $n$. According to the Ref. 8 the latter supports $|n|$ zero modes. Thus, the zero modes associated with $n$ individual widely separated vortices transform smoothly into $n$ zero modes of the $n$-fold vortex.

The vortex-antivortex configuration, by contrast, does not have exact zero modes. Now the two bound states reside on different sublattices, Fig. 3 ( c), so there is a finite tunneling amplitude between them. This yields two midgap states with energies $\pm E_{\mathrm{va}}$ that depend exponentially on the vortex-

(a)

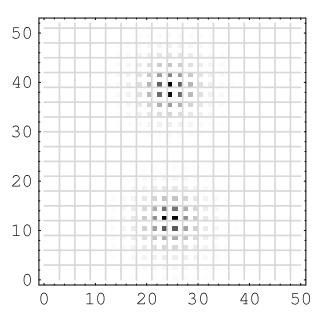

(c)

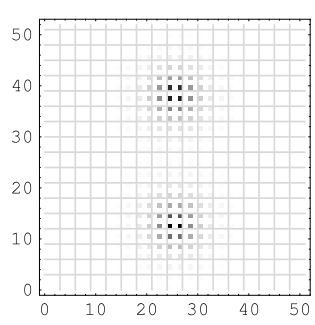

(b)

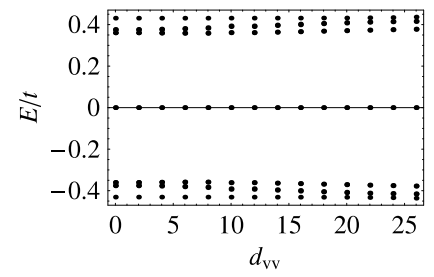

(d)

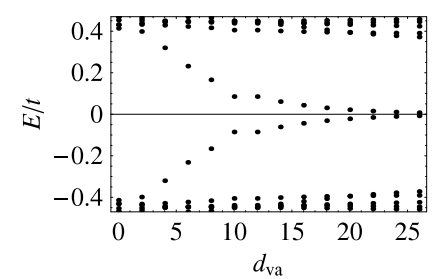

FIG. 3: The charge density at half-filling with both midgap states filled $(\mathrm{a}, \mathrm{c})$ and the low-energy (including midgap) eigenvalues $(\mathrm{b}, \mathrm{d})$ of a system with two $U(1)$ vortices $(a, b)$ and with a vortex and an antivortex (c,d). The gap $m=0.2$, the lattice size is $52 \times 51$ and the gridline spacing is 3 .

antivortex separation $d_{\mathrm{va}}$ and merge with the continuum states as $d_{\mathrm{va}} \rightarrow 0$, Fig. 3(c). Again, these results are consistent with the index theorem ${ }^{9}$ since the total vorticity of a vortexantivortex pair vanishes.

Peierls distortion.- - In a crystalline system the dimerizations postulated in our model may occur through a Peierls distortion of the ion lattice in the presence of the magnetic flux at or near half-filling. Such a distortion is driven by the electron kinetic energy gain upon the opening of a gap. We briefly outline this mechanism. At half filling, the energy gain per site is $\delta \mathcal{E}_{e}=-\frac{4 t}{N} \sum_{\mathbf{k}}\left[E_{\mathbf{k}}(\mathbf{m})-E_{\mathbf{k}}(0)\right]$, where $N$ is the number of sites and $E_{\mathbf{k}}(\mathbf{m})$ is given by the positive branch of Eq. (4). This gain is to be balanced against the loss of elastic energy that results from the ion displacements $\mathbf{u}_{i}, \delta \mathcal{E}_{\text {latt }}=\frac{K}{2 N} \sum_{\langle i j\rangle}\left(\mathbf{u}_{i}-\mathbf{u}_{j}\right)^{2}$. We assume henceforth that the change in the hopping element is related to the displacement as $m_{i, i+\hat{\mu}}=g \hat{\mu} \cdot\left(\mathbf{u}_{i}-\mathbf{u}_{i+\hat{\mu}}\right)$ with $g$ the dimensionless electron-phonon coupling $\frac{13}{\underline{13}}$ For the dimerization pattern characterized by a constant, spatially uniform vector $\mathbf{m}$, we have $\delta \mathcal{E}_{\text {latt }} / 4 t=\frac{1}{2} \kappa m^{2}$ with $\kappa=K / 4 t g^{2}$.

The Peierls distortion occurs when $\delta \mathcal{E}=\delta \mathcal{E}_{e}+\delta \mathcal{E}_{\text {latt }}$ becomes negative. Unlike the one-dimensional case, where the Peierls instability occurs at infinitesimal $g$, in two dimensions $g$ must exceed a critical value. In terms of $\kappa$ this happens for $\kappa<\kappa_{c}=N^{-1} \sum_{\mathbf{k}} \sin ^{2} k_{x} / E_{\mathbf{k}}(0) \simeq 0.806$. Near the transition we can expand

$$
\frac{\delta \mathcal{E}}{4 t}=\frac{1}{2}\left(\kappa-\kappa_{c}\right) m^{2}+\frac{2}{3 \pi}|m|^{3}+O\left(m^{4}\right) .
$$

We observe that the leading terms in $\delta \mathcal{E}$ are isotropic in $\mathbf{m}$. The anisotropy first enters at the fourth order via a term $\sim\left(m_{x}^{2}-m_{y}^{2}\right)^{2}$. This indicates that for small $m$ a vortex in this model will closely resemble the U(1) vortex in Fig. 1(c), with a small four-fold anisotropy. For $m \simeq 1$ the anisotropy be- 
comes important and the pattern will be closer to a $Z_{4}$ vortex, Fig. 1(d).

These findings are important since the $Z_{4}$ vortices are generically confined by a linear potential due to the domain walls while the U(1) vortices are not. For the fractional charges to be observable we require that the $Z_{4}$ confinement length scale be much larger than $\xi$. It is also possible that the U(1) symmetry emerges universally at the dimerization transition. We note that in this case the small $Z_{4}$ anisotropy of the present model is an irrelevant perturbation while the $Z_{3}$ in graphene ${ }^{4}$ is relevant.

Experimental realization.-In a natural solid with lattice constant $a_{0} \approx 2 \AA$ the magnetic field required to achieve the $\Phi_{0} / 2$ flux per plaquette is of the order $10^{4} \mathrm{~T}$ and thus much too high to reach in a laboratory. A better chance for observing the phenomena discussed here is to artificially engineer the hosting system such that the electrons move in a lattice with much larger $a_{0}$.

Indeed several schemes have been proposed and implemented recently to achieve this effect in semiconductor heterostructures (such as GaAs/AlGaAs) combined with a type-II superconducting layer ${ }^{3,14}$ or a top gate realized by the diblock copolymer nanolithography technique. ${ }^{15}$ A second possibility is offered by fermionic cold atoms in an optical lattice with artificial magnetic flux. ${ }^{16}$ Once the tight binding model with the magnetic flux is realized by any of the above techniques there of course remains the challenge of creating a vortex and detecting the fractional charge.
Conclusion.-We showed through general arguments and a detailed numerical study that the system proposed here supports configurations with fractional charge while preserving time-reversal symmetry. The number of zero modes in vortex configurations leading to this fractionalization is topologically protected. This should be contrasted with similar zero modes in vortices of a chiral $p$-wave superconductor where such a generic topological protection seems not to exist. ${ }^{17}$ An interesting question is what exchange statistics these fractional particles obey. This is significant theoretically as well as for potential applications in quantum information processing. A natural expectation, based on the analogy with the fractional quantum Hall states, $\stackrel{1,2}{,}$ is that they are anyons. This is also suggested by arguments using gauge invariance on a torus. 18 Since the system is time-reversal invariant one expects the effective theory for the topological excitations to be a "doubled" Chern-Simons gauge theory of the type discussed in Ref. 19. Indeed, our preliminary investigation ${ }^{20}$ suggests that such a gauge structure will emerge upon integrating out the Fermi fields in the Dirac Hamiltonian (5) in the presence of topologically nontrivial background field $\chi$.

Acknowledgment.-The authors have benefited from discussions and correspondence with L. Balents, M.P.A. Fisher, V. Gurarie, R. Jackiw, K. Madison, G. Rosenberg, J. Pachos and A. Vishwanath. This work has been supported by the CIAR, NSERC, NSF Grant No. PHY05-51164 (KITP) and the Killam Foundation.
1 R. B. Laughlin, Phys. Rev. Lett. 50, 1395 (1983).

2 D. Arovas, J. R. Schrieffer, and F. Wilczek, Phys. Rev. Lett. 53, 722 (1984).

${ }^{3}$ C. Weeks, G. Rosenberg, B. Seradjeh, and M. Franz, Nature Physics (in press, AOL publishing Sep 30, 2007 doi:10.1038/nphys730, arXiv:cond-mat/0703001).

${ }^{4}$ C.-Y. Hou, C. Chamon, and C. Mudry, Phys. Rev. Lett. 98, 186809 (2007).

5 J. Goldstone and F. Wilczek, Phys. Rev. Lett. 47, 986 (1981).

6 W. P. Su, J. R. Schrieffer, and A. J. Heeger, Phys. Rev. Lett. 42, 1698 (1979).

7 R. Jackiw and S.-Y. Pi, Phys. Rev. Lett. 98266402 (2007).

${ }^{8}$ R. Jackiw and P. Rossi, Nucl. Phys. B 190, 681 (1981).

9 E. J. Weinberg, Phys. Rev. D 24, 2669 (1981).

${ }^{10}$ R. Jackiw and C. Rebbi, Phys. Rev. D 13, 3398 (1976).

11 M. Levin and T. Senthil, Phys. Rev. B 70, 220403(R) (2004).
12 N. Read and S. Sachdev, Phys. Rev. B 42, 4568 (1990).

13 S. Tang and J. E. Hirsch, Phys. Rev. B 37, 9546 (1988). See also Y. Ono and T. Hamano, J. Phys. Soc. Japan 69, 1769 (2000).

14 M. Berciu, T. G. Rappoport, and B. Janko, Nature 435, 71 (2005).

15 S. Melinte et al., Phys. Rev. Lett. 92, 036802 (2004).

16 D. Jaksch and P. Zoller, New J. Phys. 5, 56 (2003); E. J. Mueller, Phys. Rev. A 70, 041603(R) (2004); A. S. Sørensen, E. Demler, and M. D. Lukin, Phys. Rev. Lett. 94, 086803 (2005).

17 V. Gurarie and L. Radzihovsky, Phys. Rev. B 75, 212509 (2007).

18 Y.-S. Wu, Y. Hatsugai, and M. Kohmoto, Phys. Rev. Lett. 66, 659 (1991).

19 M. Freedman, C. Nayak, K. Shtengel, K. Walker and Z. Wang, Ann. Phys. 310428 (2004).

${ }^{20}$ B. Seradjeh and M. Franz, (unpublished). 